\title{
Gelungene und mißlungene Kanonisierung: Dantes Commedia und Klopstocks Messias
}

\author{
Matías MaRTínez (München)
}

Über die Kanonisierung eines literarischen Werkes entscheidet nicht sein Autor, sondern die Nachwelt. Gelegentlich ist jedoch bereits die Produktion durch kanonischen Geltungsanspruch geprägt. Dantes Commedia und Klopstocks Messias sind besonders markante Beispiele fur Werke, die als kanonische nicht erst rezipiert, sondern bereits konzipiert wurden. Ein Vergleich von Dante und Klopstock kann sich allerdings nicht auf einen direkten Rezeptionszusammenhang berufen. Klopstock hat Dantes Werk erst kurz vor AbschluB des Messias kennengelernt und offenbar nicht geschätzt; seine Pfortenser Abschiedsrede über das Epos (zu einer Zeit, als Klopstock den Grundriß des Messias festlegte) erwähnt Dantes Namen nicht einmal. ${ }^{1}$ Es geht hier vielmehr um einen kontrastiven Vergleich zweier Werke, die im Selbstverständnis ihrer Autoren, im Erzählstoff, in der literarischen Gestaltung und auch in der unmittelbaren Aufnahme durch die Zeitgenossen bemerkenswerte Gemeinsamkeiten aufweisen, deren spätere Rezeption aber diametral auseinanderläuft in die heutigen Extrempositionen eines Klassikers par excellence und eines nur noch versprengten Fachleuten zugemuteten Archivstïcks. Wie haben diese Autoren ihren kanonischen Geltungsanspruch textintern und paratextuell inszeniert? Warum wurde Dantes Werk auf Dauer im Kanon etabliert, Klopstocks hingegen nicht?

Meine (gewiß nicht vollständigen) Antworten auf diese Fragen lassen sich vorweg in drei Thesen zusammenfassen:

- Die selbstinszenierte Kanonisierung erfolgt bei Dante wie bei Klopstock im Rahmen einer religiös fundierten Poetik, in der das Konzept einer fiktionalen Literatur keinen Platz hat.

- In beiden Fällen wird die religiöse Legitimationsstrategie jedoch durch den Einsatz fiktionaler Elemente zirkulär und damit theologisch prekär.

- Das Gelingen bzw. Mißlingen einer dauerhaften Kanonisierung ist im unterschiedlichen Potential beider Werke begründet, unter den veränderten Bedingungen eines modernen, autonomen Literaturbegriffs rezipierbar zu bleiben.

1 Zum Verhältnis Klopstocks zu Dante vgl. Gerhard Kaiser, Klopstock. Religion und Dichtung, 2. durchges. Aufl., Kronberg/Ts. 1975, 204 f. Zu Klopstocks Pfortenser Rede siehe unten Anm. 29 und 31. 
Dantes teils exzessive, teils paradoxe Erfullung der Ansprüche einer theologisch dominierten mittelalterlichen Poetik war hier erfolgreicher als Klopstocks vier Jahrhunderte jüngerer Versuch einer »heiligen Poesie«. Ein Grund dafür könnte in der Erzählweise der Commedia liegen, die eine fiktionalisierende sextensiver Lektïre gestattete, während Klopstocks >Dunkelheit< in der Moderne nurmehr in der Lyrik, nicht aber in langen narrativen Texten durchsetzbar war.

I.

Zunächst zu Dante. Die Commedia, das früheste Werk des neuzeitlichen literarischen Kanons, ist ein Schwellentext, in dem sich mittelalterliche und neuzeitliche Kriterien für Literatur und literarische Kanonbildung mischen. Ich möchte das an drei Aspekten verdeutlichen. Sie betreffen die Sprecherinstanz (Autor/Erzähler), die Erzählstruktur (erzählendes vs erlebendes Ich) und die intendierte und faktische Rezeption durch die Zeitgenossen.

1.) Sprecherinstanz: Dante inszeniert sich in der Commedia in ungewöhnlich starkem MaB als Autor, aber zugleich entwertet er den Status des Autors. Ich beginne mit dem offensichtlichen Umstand, daB die Commedia eine Ich-Erzählung ist: Es besteht eine Identität zwischen dem Autor, dem Erzähler und dem Protagonisten des Textes. Wie Augustinus in den Confessiones, aber in krassem Unterschied zur epischen Tradition und insbesondere zu Vergils Aeneis, erzählt Dante von sich selbst. Das äßBert sich nicht nur in der Ich-Form und der Verwendung seines eigenen Namens (er nennt ihn allerdings nur einmal und legt ihn nicht sich selbst, sondern Beatrice in den Mund, siehe Pur. 30,55, vgl. Pur. 30,63), ${ }^{2}$ sondern auch in den zahlreichen Referenzen auf die Biographie des Autors, die insbesondere in den Begegnungen mit verstorbenen Freunden, Lehrern, Bekannten und Vorfahren hergestellt werden. Und nicht zuletzt ist es die Figur der Beatrice, welche - wie immer man ihren historischen Status auch einschätzt - die in der Commedia erzählte Geschichte mit der Vita Nuova und dem Convivio und damit über den Textrahmen der Commedia hinaus mit anderen Werken des realen Autors Dante (und nicht nur mit dem immanenten Ich-Erzähler der Commedia) verklammert. Wegen dieser autobiographischen Klammer von Autor, Erzähler und Held gibt es in der Commedia keine Verdoppelung der Autorfunktion wie in fiktionalen Texten, in denen die (fiktive) Rede des Erzählers von der (fiktionalen) Rede des Autors zu unterscheiden wäre. ${ }^{3}$

In Dantes Selbstinszenierung als Autor äußert sich ein für seine Zeit außergewöhnliches Selbstbewußtsein, das für das eigene Werk kanonische Geltung be-

2 Dante Alighieri, La Commedia secondo l' antica vulgata, hrsg. Giorgio Petrocchi, Mailand 1975. Nach dieser Ausgabe wird im folgenden zitiert.

3 DaB die Wahmehmung von Fiktionalität durchaus im allgemeinen literarischen Verständnisspielraum der Zeit lag, beweisen bereits, mehr als ein Jahrhundert vor Dante, die klassischen Artusromane Chrétiens und Hartmanns mit ihren deutlichen Fiktionalitätssignalen. Allerdings sind stoff- und gattungsspezifische Unterschiede zu berïcksichtigen. 
ansprucht. Im 4. Gesang des Inferno setzt Dante sich mit den fünf »poete« der »bella scola« Homer, Vergil, Horaz, Ovid und Lukan gleich (Inf. 4,94/100-102)4 und weist sich selbst so, als >poeta<, einen Titel zu, den Statius im Purgatorio zum »nome che più dura e più onora« (Pur. 21,85) erklärt.

Verdeckter, doch noch ehrgeiziger ist Dantes Selbsterhebung zum sscribar - ein seltener poetologischer Terminus, der in der Commedia nur einmal vorkommt (Par. 10,26f.). 5 In De Monarchia verwendet Dante sscribar zweimal, stets als Bezeichnung für göttlich inspiriertes Schreiben: Der Evangelist Lukas wird "scriba Cristi« genannt (Mon. II,VIII,14), ${ }^{6}$ und allgemeiner heiBt es: "Nam quanquam scribe divini eloquii multi sint, unicus tamen dictator est Deus, qui beneplacitum suum nobis per multorum calamos explicare dignatus est« (Mon. III,IV,11). Damit nimmt Dante für die Bestimmung des sscribar die Lehre von der Verbalinspiration der biblischen Texte in Anspruch. Wenn er sich in der Commedia selbst als sscribar bezeichnet, dann erscheint er nicht als autonomer Autor seines Textes, sondern wie die normale Bedeutung von sscribar, >Sekretär<, bereits nahelegt - als Medium göttlicher Inspiration, als prophetischer poeta vates. ${ }^{7}$ Diese Selbstlegitimation durch göttliche Inspiration stellt eine in mittelalterlicher Literatur wohl einzigartige Aufwertung der eigenen Produktion dar, ebenso einzigartig wie die Bezeichnung der Commedia als »poema sacrok (Par. 25,1).

Die Verschiebung von poetischem zu sakralem Anspruch ist auch an den Invokationen der Commedia abzulesen. ${ }^{8}$ In jeder der drei Cantiche wendet Dante sich an übergeordnete Instanzen mit der Bitte um Hilfe bei der Gestaltung des Stoffes, wobei die Reihe der Invokationen eine Steigerung von topisch-rhetorischem zu sakralem Gehalt aufweist. Ruft Dante zunächst die Musen, Apollo, Minerva und Abstrakta wie den Geist (wmente«) oder die Kunstfertigkeit (wingegno«) an, so tritt am Ende des Werkes der religiöse Anspruch unverhüllt hervor, wenn für die Schilderung des Empyreums im zehnten und letzten Himmel und für die abschließende Gottesschau Gott direkt um Hilfe gebeten wird (Par. 30,97-99; 33,67-72).9

4 Zum Selbstverständnis Dantes als Autor vgl. die Zusammenfassung von August Buck, *Die Commediax, in: Dantes Commedia und die Dante-Rezeption des 14. und 15. Jahrhunderts, GRLMA X/1, Heidelberg 1987, 21-165, hier: 142-153. Dante fuihrt in der Commedia neben dem literarischen nicht nur einen religiösen, sondern auch einen politischen und einen wissenschaftlichen Diskurs; zu letzterem vgl. Willi Hirdt, »Künstler und Wis-

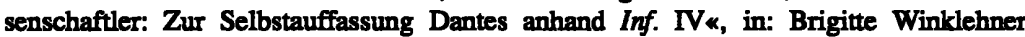
(Hrsg.), Literatur und Wissenschaft. Begegnung und Integration, Festschrift für Rudolf Baehr, Tübingen 1987, 255-271.

5 Vgl. Gian Roberto Sarolli, Prolegomena alla sDivina Commediar, Florenz 1971, Kap. 2: »Dante scriba Deik, bes. 233-246.

6 Monarchia, Le Opere di Dante Alighieri, V, hrsg. Pier Giorgio Ricci, o. O. 1965.

7 Vgl. Raoul Manselli, «Profetismo«, Enciclopedia Dantesca IV, 2. Aufl., Rom 1984, Sp. 694-699.

$8 \mathrm{Zu}$ den Musen vgl. Ernst Robert Curtius, Europäische Literatur und lateinisches Mittelalter, 10. Aufl., Bern, München 1984, 235-252, bes. 245 f.; speziell zu Dante vgl. Buck (Anm. 4), 143-145.

9 Im Brief an Cangrande erklärt Dante allerdings bereits die heidnisch-rhetorischen Figuren des Apollo, der Minerva und der Musen zu Instanzen einer sgleichsam göttlichen Gaber (»quasi divinum munusk) und zu Gewährleistern göttlicher Inspiration, die den Dichter 


\section{Matías Martínez}

2.) Erzählstruktur: Anders als sein episches Vorbild Vergil schildert Dante kein kollektives Geschehen, kein historisch bedeutsames und identitätstiftendes Gründungsereignis seiner kulturellen Gemeinschaft, sondern eine insgesamt durch keine auctoritas beglaubigte individuelle Reise durch die Reiche des Jenseits. Kollektiv verbindlich wird diese Reise durch ihre mit der L̇ehre vom mehrfachen Schriftsinn verknüpfte allegorische Auslegung, worauf ich hier nicht eingehe. ${ }^{10}$ Aber auch in anderer Weise wird die individuelle Geschichte der Hauptfigur Dante in ein überindividuelles Ganzes transponiert. Hans Robert Jauß wies kürzlich darauf hin, daB der Autor Dante »das individuelle Schicksal des Wanderers am Ende in der Gemeinschaft der Heiligen aufgehen läßt«. ${ }^{11}$ Denn der christliche Dichter, so Jauß, müsse »sein fragmentarisches Leben auf den Endhorizont eines Glaubens öffnen, der alles Zeitliche und Individuelle uibersteigt« (176). Die offene Erzählstruktur einer Wanderung wird in der Commedia überlagert durch die zielgerichtete Ausrichtung des Geschehens auf die Ankunft im höchsten Ort des Weltalls, dem Empyreum. Die Handlung ist aber nicht nur zielgerichtet, sondern auch zukunftsgewiB. Denn daß Dantes Wanderung das gesetzte Ziel auch erreichen wird, daran lassen die Voraussagen Vergils vom ersten Gesang der Commedia an keinen Zweifel - auch wenn Dantes erlebendes Ich, das an seinen offenen, zukunftsungewissen Erlebnishorizont gebunden ist, immer wieder um den Erfolg der Wanderung bangt. Der Leser/Hörer weiß es, belehrt durch das erzählende Ich, besser. Dantes Weg ist »fatale« (Inf. 9,97), ist vorherbestimmt. Das dargestellte Geschehen der Commedia, das sich im Erleben des Wanderers Dante vor dem offenen Horizont einer unsicheren $\mathrm{Zu}$ kunft abspielt, liegt in Wahrheit von Beginn an fest, es ist >von hinten motiviert< (Clemens Lugowski); ${ }^{12}$ dem Zeitstrom der Ereignisse ist das Offene, Ungewisse genommen, er enthüllt sich unter dem Blick göttlichen Allwissens, dem die menschliche Geschichte vollständig vorliegt, als bloße Oberfläche einer fundamentalen, zeitlosen, absolut gesicherten Ordnung: "La contingenza ... tutta è dipinta nel cospetto etterno ( Par. 17,37/39).

Aber Dantes Mission ist ja mit der Gottesschau im letzten Gesang der Commedia nicht beendet. Zwar fällt das Ende der Erzählung (discours) mit dem Ende der erzählten Geschichte (histoire) zusammen. Doch Dante muß noch zur Erde zurückkehren und den Lebenden seine Visionen zum Zwecke moralischer Läuterung mitteilen. Der Text der Commedia ist das Ergebnis dieser Mission. >Dante< erfillt die doppelte narrative Funktion einer erlebenden Figur und einer

vor den übrigen Menschen auszeichne (»contra comunem modum hominem«); vgl. Dante Alighieri, Das Schreiben an Cangrande della Scala, lat.-dt., übers., eingel. u. komm. Thomas Ricklin, Hamburg 1993, § 47 (im folgenden zitiert als «Ep. XIII« mit Paragraphenzahl).

10 Vgl. dazu meinen Aufsatz „Dante und der Ursprung des Kanons«, in: Maria Moog-Grïnewald (Hrsg.), Kanon und Theorie, Heidelberg 1997, 139-152.

11 Hans Robert Jauß, „Erleuchtete und entzogene Zeit - eine Lectura Dantis«, in: ders., Wege des Verstehens, München 1994, 147-180, hier: 147.

12 Vgl. Clemens Lugowski, Die Form der Individualität im Roman, Frankfurt a. M. 1976, 66-81. 
Erzählerinstanz. Während die Geschichte des erzählten Ich mit der Gottesschau endet, beginnt diejenige des erzählenden Ich erst mit der Rückkehr auf die Erde. Die Geschichte von Dantes Wanderung, die uns in der Commedia mitgeteilt wird, ist zugleich die Vorgeschichte des Erzählens dieser Geschichte.

Erzähltes und erzählendes Ich, histoire und discours der Commedia geraten so in ein paradoxes wechselseitiges Fundierungsverhältnis. Einerseits fundiert die Geschichte des Wanderers Dante den Geltungsanspruch des Autors Dante: nur weil er die unerhörten Reiche des Jenseits bis hin zur Gottesschau erfahren hat, darf Dante ein "poema sacro« (Par. 25,1) schreiben. Andererseits legitimiert der reale Autor Dante - wie wir gleich sehen werden - die Geschichte vom Wanderer Dante mit der moralischen Wirkungsabsicht. Einerseits ist die histoire die metaphysische Basis des discours; andererseits ist sie seine didaktisch-rhetorische Hervorbringung. ${ }^{13}$

Indem Dante für die Commedia die Ich-Form wählt und dadurch zugleich Hauptfigur und Autor seiner Geschichte wird, paradoxiert er den theologischen Geltungsanspruch seines Werkes. Eine Auflösung dieser Paradoxie wäre erst unter den Bedingungen eines fiktionalen Literaturbegriffs möglich, wonach das erzählende Ich als fiktive Sprecherinstanz vom realen Autor abzugrenzen wäre. Dann erst könnte das erzählende Ich die Legitimation seines discours aus der histoire ziehen, während der Autor die Legitimation der histoire über den Wirkungsanspruch des discours begründet.

3.) Intendierte und faktische Rezeption: Dante inszenierte nicht nur, wie gezeigt, innerhalb des Textes der Commedia seine eigene Kanonisierung, sondern versuchte auch auf anderen Wegen, die Rezeption entsprechend zu steuern. Er trat nicht nur als auctor, sondern auch - und das ist in diesem Umfang für seine Zeit durchaus ungewöhnlich - als commentator der Commedia in Erscheinung. ${ }^{14}$ Im Schreiben an Cangrande erklärt Dante sein Werk zum »doctrinale opus« (Ep. XIII, \& 18), das der moralischen Unterweisung diene: „Genus vero phylosophie sub quo hic in toto et parte proceditur, est morale negotium, sive ethica; quia non ad speculandum, sed ad opus inventum est totum et pars« (Ep. XIII, \& 40). Das Ziel dieser moralischen Unterweisung sei, die Lebenden aus dem Elend herausund dem Glück zuzuführen: »finis totius et partis est removere viventes in hac vita de statu miserie et perducere ad statum felicitatis« (Ep. XIII, § 39). Diese allegorische Interpretation mit moralisch-didaktischer Wirkungsabsicht wird in ihrem Anspruch erheblich verschärft durch die für profane Texte ungebräuchliche Anwendung der Lehre vom vierfachen Schriftsinn (die nach orthodoxer Auf-

13 Ich übertrage hier eine Beobachtung Rainer Warnings über Dantes Amortheologie in der Vita Nuova, vgl. Rainer Warning, wImitatio und Intertextualität. Zur Geschichte lyrischer Dekonstruktion der Amortheologie: Dante, Petrarca, Baudelairex, in: Willi Oelmüller (Hrsg.), Kolloquium Kunst und Philosophie, II: Ästhetischer Schein, Paderborn 1982, 168-207, hier: 171.

$14 \mathrm{Zu}$ Dante als Kommentator und den Commedia-Kommentaren vgl. Bruno Sandkühler, Die frühen Dantekommentare und ihr Verhältnis zur mittelalterlichen Kommentartradition, München 1967, und ders., »Die Kommentare zur >Commediax bis zur Mitte des 15. Jahrhunderts火, in: GRLMA X/1 (Anm. 4), 166-208. 
fassung der Auslegung der heiligen Schrift vorbehalten war); Dante scheint sie in einer (nicht ganz deutlichen) Passage des Schreibens an Cangrande auf sein eigenes Werk zu beziehen (vgl. Ep. XIII, §§ 20-22).15 Die zahlreichen Kommentare und öffentlichen Auslegungen, die nach Dantes Tod einsetzten, folgten der vom Autor vorgezeichneten gelehrten Interpretation des Werkes.

Doch gab es auch gegenläufige Rezeptionstendenzen, die Dantes Vorgaben zuwiderliefen. Das Verbot von Dantes volkssprachlichen Werken einschlieBlich der Commedia, welches das Florentiner Provinzialkapitel der Dominikaner 1335 erließ, wurde mit dem Wunsch begründet, die Brüder sollten sich stärker dem Studium der Theologie widmen (wut fratres nostri ordinis theologie studio plus intendant $\ll)^{16}$ - offenbar sahen die Dominikaner theologische Materien in der Commedia nicht angemessen behandelt. Insbesondere der ontologische Status von Dantes Jenseitsreise muB für orthodoxe Zeitgenossen ein Problem dargestellt haben - und das unabhängig von der Frage, wie Dantes eigene Interpretation seines Werkes anhand des mehrfachen Schriftsinns im Schreiben an Cangrande zu verstehen ist. Auffällig ist jedenfalls, daB die frühen Kommentatoren der Commedia, wohl um Häresievorwürfe abzuweisen, zur »Untermauerung der Rechtgläubigkeit «17 immer wieder betonen, das dargestellte Geschehen sei bloB in allegorischem, nicht in literalem Sinn zu verstehen.

Bedenkt man die große Faszination, welche die damals weitverbreitete Visionsliteratur beim ungelehrten Publikum genoB, und berücksichtigt man die mentalitätsgeschichtliche Tatsache, daB »das Mittelalter durch ein ganz intensives Bedürfnis nach dem Eingriff des Überweltlichen gekennzeichnet ist « ${ }^{18}$, dann ist es wenig wahrscheinlich, daß die ungelehrte Rezeption der Commedia - von der wir wegen der großen und sozial gestreuten Verbreitung der Manuskripte zwar wissen, daB sie stattgefunden hat, aber nicht, wie ihr Textverständnis beschaffen war - der offiziösen Lesart im Sinne Dantes gefolgt ist. Vielleicht dürfen wir uns hier von der bekannten Anekdote leiten lassen, die Boccaccio um 1360 in seinem Tratatello in laude di Dante erzählt:

Dante sah dunkel aus, mit dichtem, schwarz gekräuseltem Haar und Bart, seine Haltung war stets traurig und gedankenverloren. Eines Tages in Verona ging er an einigen Frauen vorbei, von denen eine zu den anderen sagte: sSeht ihr den Mann dort, der in die Hölle hinabsteigt und zurückkehrt wie es ihm gefallt, und uns Neuigkeiten von denen da unten bringt? Darauf antwortete eine andere Frau: ,Was du sagst, muB tatsächlich wahr sein, denn sein Bart ist gekräuselt und sein Antlitz geschwärzt von der Hitze und dem Rauch dort untens. 19

15 Vgl. Sandkiihler, «Die Kommentare zur >Commedia«« (Anm. 14), 167, und Ricklins Kommentar zu Ep. XIII (Anm. 9), \$\$ 20-22, 70-80.

16 Zitiert nach Dante, Schreiben an Cangrande (Anm. 9), »Einleinung«, XVII.

17 Sandkuihler, »Die Kommentare zur >Commediar« (Anm. 14), 171.

18 Peter Dinzelbacher, Vision und Visionsliteratur im Mittelalter, Stuttgart 1981, 59.

19 Giovanni Boccaccio, Opere, hrsg. Pier Giorgio Ricci, Mailand 1965, 608 (meine Übersetzung). Boccaccio macht allerdings anschließend deutlich, daß er dieses Textverständnis für naiv hält. 
Worauf es mir in unserem Zusammenhang ankommt, ist, daß keine der drei skizzierten Rezeptionslinien, weder die allegorische der Kommentatoren noch die Häresie vermutende der Dominikaner noch auch die (hier nur anekdotisch illustrierte) des ungelehrten Publikums, die Commedia als fiktionalen Text gelesen hat. Entweder wurde die erzählte Welt allegorisch und moralisch-didaktisch interpretiert, so daB die Fiktion lediglich Hilfscharakter hatte und unmittelbar im Dienst außerliterarischer Darstellungsziele stand - oder aber sie wurde als real aufgefaßt.

II.

In einem Sprung über mehr als vier Jahrhunderte wende ich mich nun Klopstocks Messias zu. Wie schon bei Dante ist auch bei Klopstock der Versuch einer Selbstkanonisierung zu beobachten, der zunächst auch überaus erfolgreich war, auf Dauer jedoch scheiterte. 1748 veröffentlichte Klopstock die ersten drei Gesänge des Messias. Im selben Jahr entstand die Ode Die Stunden der Weihe, die sich auf das Messias-Projekt bezieht und in gedrängter Form dieselben Strategien einer Selbstkanonisierung aufweist, die Klopstock auch in seinem Epos verwendet. Sie kann uns deshalb hier als Zugang dienen. Die ersten fünfeinhalb der insgesamt elf Strophen lauten:

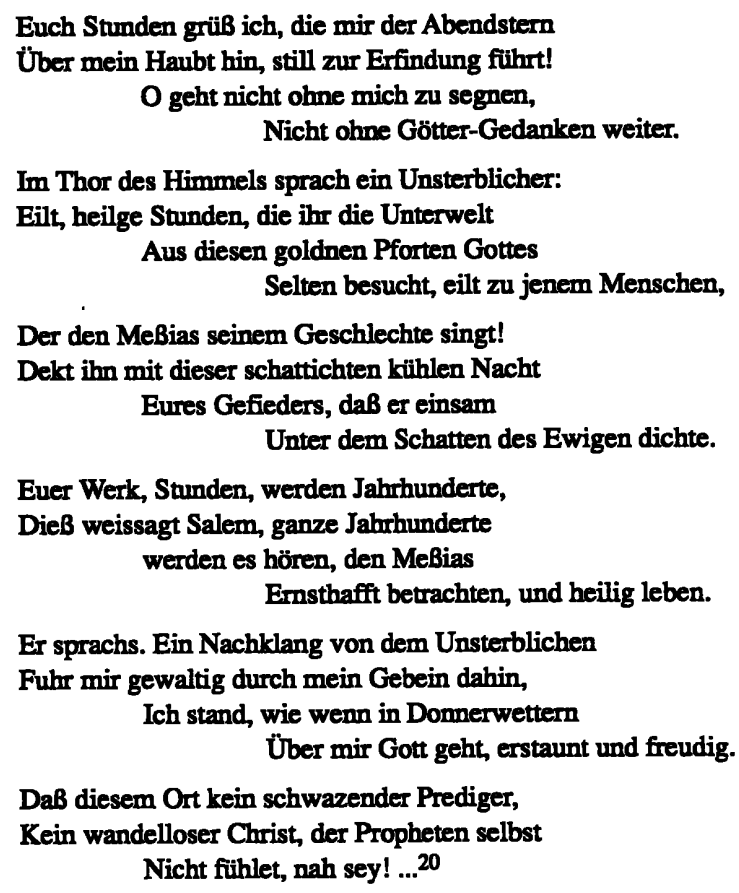

20 Ich zitiere die erste erhaltene Fassung, abgedruckt in Friedrich Gottlieb Klopstock, Der Messias. Gesang I-III, hrsg. Elisabeth Höpker-Herberg, Stuttgart 1986, 110. 
In diesen Odenstrophen werden Geltungsanspruch und Wirkungsabsicht auch des Messias formuliert. Der übergeordnete Sprecher der Verse (der in den Strophen 2 bis 4 einen »Unsterblichen«, nämlich den Seraphen Salem, in direkter Rede zu Wort kommen läBt) ist ein von den Abendstunden besuchter Mensch, dessen Identität mit dem Autor aus dem Projekt, den »MeBias« zu singen, hervorgeht (vgl. v. 9). Nicht um ein Rollengedicht handelt es sich hier, sondern um eine programmatische Selbstinszenierung Klopstocks. Der Dichter legitimiert das kühne Unternehmen, durch seinen Gesang künftigen Jahrhunderten das »heilig leben« zu ermöglichen, mit der Berufung auf die personifizierten Abendstunden, die als »heilge« Stunden der Weihe ihrerseits in himmlischem Auftrag handeln. Er erscheint als bevorzugtes (»einsam«), göttlich inspiriertes Medium, dessen Rede »unter dem Schatten des Ewigen« von den Abendstunden geboren wird. Das Eintreten der erwünschten Wirkung wird in zukunftsgewissem Futur von der göttlichen und damit unfehlbaren Instanz des seraphischen Salem geweissagt.

Betrachtet man die Sprechsituation der Ode jedoch genauer, so zeigt sich eine eigentïmlich gebrochene, ja zirkuläre Strategie der Selbstlegitimation. Zwar wird der Geltungsanspruch der poetischen Rede zurückgeführt auf die Worte des Salem, Worte, von denen im indikativischen Präteritum berichtet und die in direkter Rede vom Sprecher-Ich zitiert werden. Aber die Kraft dieser Beglaubigung der poetischen Rede ist in dreierlei Hinsicht eingeschränkt. Erstens ist der Seraph Salem keine durch den biblischen Text belegte Instanz, sondern eigens von Klopstock erfunden. Zweitens empfängt der Dichter seinen himmlischen Auftrag nicht direkt durch den ïbersinnlichen Seraphen, sondern sinnlich vermittelt durch die Natur in Gestalt der Abendstunden. Drittens legt die übergreifende Tempusgestaltung der Ode es nahe, den überirdischen Ursprung der dichterischen Rede zugleich auch als deren Produkt zu verstehen: In der einleitenden ersten Strophe bittet der Sprecher im Präsens, also im Tempus-Register des >Besprechens<, um »Götter-Gedanken«.21 Nach der Tradition des Musenanrufs wäre zu erwarten, daB in den nun folgenden Versen im serzählenden Tempus-Register des Präteritum eben die "Gedanken« ausgesprochen würden, für die die Muse um Hilfe gebeten worden war. Anders hier. Denn die folgenden Strophen 2 bis 5 begründen allererst den überirdischen Ursprung der dichterischen Rede; zugleich aber erscheinen sie als Produkt der zunächst präsentisch besprochenen dichterischen »Erfindung « (v.2). Wenn diese Analyse zutrifft, dann liegt in Klopstocks Ode dieselbe zirkuläre Argumentationsstruktur wie in Dantes Commedia vor, um die poetische Rede als inspirierte zu legitimieren: Der Verweis auf numinose Instanzen als Quelle der poetischen Rede weist wiederum auf den Akt der poetischen Hervorbringung zurück.

Sowohl der religiöse Geltungsanspruch wie auch die paradoxe Beglaubigung der dichterischen Rede in der Ode Die Stunden der Weihe kehren im Messias wieder. Klopstock meint, die antiken Ependichter zu übertreffen, weil er, »Kühn

$21 \mathrm{Zu}$ den >Tempusregistern > Besprechen $<$ und >Erzählen vgl. Harald Weinrich, Textgrammatik der deutschen Sprache, Mannheim 1993, 198-207. 
und erhaben, nicht singt verschwundene Größe des Menschen« $(1,584), 22$ sondern die Passion und Auferstehung Christi zum Stoff nimmt. Die einzigartige Bedeutung dieses Stoffes erhöhte auch dessen poetische Darstellung: »Du aber, Gesang von dem Mittler, ... Sieger der Zeiten, Gesang, unsterblich durch deinen Inhalt« $(15,470 / 2)$. Nach dem Tode Klopstocks sollen »Meine Freunde mein Grab mit Lorbern und Palmen umpflanzen, / Daß, wenn in himmlischer Bildung dereinst von dem Todk ich erwache, / Meine verklärte Gestalt aus stillen Hainen hervorgeh« (3,9-11). Poetischer Lorbeer, biblische Palme: dichterischer Ruhm und religiöse Erlösung werden hier gleichermaßen beansprucht. ${ }^{23}$

Der gattungstypische Musenanruf ${ }^{4}$ wird von Klopstock in Gestalt der immer wieder angerufenen »Sionitin« in christlichem Gewand aufgenommen: „Die du himmlische Lieder mich lehrst, Gespielin der Engel, / Seherin Gottes, du Hörerin hoher unsterblicher Stimmen, / Melde mir, Sionitin, das Lied, das die Engel itzt sangen.« (1,242-244; vgl. 8,1-6). So traditionell und zentral die Sionitin als Musenfigur für die Beglaubigung der epischen Rede im Messias ist, so prekär ist ihr religiöser Status. Denn wie schon bei dem Engel Salem in der Ode Die Stunden der Weihe, handelt es sich auch bei der »Sängerin Sions« $(3,12)$ nicht um eine biblisch vorgegebene Beglaubigungsinstanz. Klopstock folgt zwar mit seiner christianisierten Muse dem Vorbild Miltons, der in Paradise Lost ebenfalls eine himmlische Muse anruft (wheavenly Muse« [I,6] bzw. „Urania« [VII,1]). Doch die einzig mögliche alttestamentarische Referenzstelle (Spr. Sal. 8) ist zu . undeutlich, als daß sie die christianisierte Musenfigur des Messias-Sängers biblisch rechtfertigen könnte. 25

Auch die in der Ode beobachtete Einschaltung von Vermittlungsinstanzen zwischen himmlischer Inspirationsquelle und irdischem Medium, welche die unmittelbare religiöse Beglaubigung der dichterischen Rede abschwächt, kehrt im Messias wieder. Anders als im antiken Epos oder bei Klopstocks unmittelbarem Vorbild Milton wird in der invocatio zu Beginn des Messias - »Sing, unsterbli-

22 Zitate aus dem Messias mit Gesang- und Verszahl nach Friedrich Gottlieb Klopstock, Der Messias, Werke und Briefe, hist.-krit. Ausg., Werke IV, Bd. 1/2: Text (Fassung von 1799/1800), hrsg. Elisabeth Höpker-Herberg, Berlin, New York 1974.

23 Hamel übersieht diese Bedeutung, wenn er kommentient: «Sonderbar mutet bei Klopstock der Wunsch nach diesen exotischen Gewächsen an« (Klopstock, Werke, Erster Teil: Der Messias, hrsg. R[ichard] Hamel, Kürschners Deutsche National-Litteratur 46, Berlin, Stuttgart 0. J., I, 128). Zur Palme als wdas zentrale Emblem des Messias schlechthin火 vgl. Jöm Dräger, Typologie und Emblematik in Klopstocks sMessiase, Diss. Göttingen 1971, 146-179, hier: 178.

24 Vgl. Curtius (Anm. 8), 235-252 (über Klopstock: 250), sowie Dieter Martin, Das deutsche Versepos im 18. Jahrhundert. Studien und kommentierte Gattungsbibliographie, Berlin, New York 1993, 103-113 (mit einer Auflistung der Musenannufe und der Exordialtopik im Messias, zusammen mit einer von mir abweichenden Interpretation).

25 Hamel kommentiert den von Klopstock erfundenen Namen »Sionitin «: „Auf Sion oder Zion wohnte der Sänger der Psalmen, David; deshalb nennt Klopstock die Himmelsmuse nach diesem Berge« (Klopstock, Werke [Anm. 23], 23). In den Fassungen bis 1755 nennt Klopstock die Musenfigur noch nicht Sionitin, sondern Muse von Tabork, »heilige« oder »unsterbliche Muse«, vgl. Richard Hamel, Klopstock-Studien, III, Rostock 1880,64 . 
che Seele, der sündigen Menschen Erlösung« (1,1) - nicht etwa die Muse, sondern die eigene Seele des epischen Sängers angerufen. Die Seele wiederum wird von verschiedenen himmlischen Instanzen wie dem »Geist Schöpfer« $(1,10)$, der sionitischen Muse (passim), dem alttestamentarischen David (8,1-4), aber auch von Christus $(10,12)$ oder Gott $(11,1-4)$ instruiert. Auch wenn nach dem Selbstverständnis des Autors die »Seele« hier als zwar subjektives, aber doch überindividuell-repräsentatives Empfangsorgan der Inspiration zu verstehen ist, ${ }^{26}$ so bleibt die Einsetzung einer fiktiven Figur als Legitimationsinstanz religiös inspirierter Rede dennoch erstaunlich. Lessing, gegenüber dem Messias durchaus positiv eingestellt, paraphrasierte diesen ersten Vers des Werkes denn auch als »Ich unsterblicher Klopstock, singe der sündigen Menschen Erlösung«27 und stellte damit dessen theologisch prekäre Sprechhaltung heraus: »Es ist auch in der Tat besonders, mit einem stolzen Ich anzufangen, und alsdann die Musen anzurufen, nachdem man schon alles auf die eignen Hörner genommen hat. Das heiBt anklopfen, wenn man die Türe schon aufgemacht hat $\ll .28$ So hat Klopstock die Beglaubigung der eigenen poetischen Rede durch Anrufe an übermenschliche Instanzen wohl auch nicht nur als konventionellen Gattungszierrat und rhetorischen Topos, sondern in einem verbindlicheren Sinne gemeint. Biographische Zeugnisse belegen, daß der Dichter sich zeitlebens als der von ihm selbst in der Pfortenser Rede beschworene "vates ${ }^{29}$ verstand, der den Impuls zum Messias als Eingebung und Vision auffaBte und sein Dichten als Bestimmung ansah. 30

Klopstock hat seinen mit dem Messias verbundenen Anspruch auf kanonische Geltung nicht nur in poetischer, sondern auch in prosaischer Rede formuliert. Drei Jahre vor der Veröffentlichung der ersten Gesänge des Messias sprach der Einundzwanzigjährige in der erwähnten Rede zum Abschied aus der Schule in Pforta über die epische Dichtkunst. Die Dichtkunst sei unter den Künsten die "vornehmste und erste Nachahmerin der Naturk und das bedeutendste Zeugnis der »Größe und Erhabenheit des menschlichen Geistes «31, sie weise eine »gewisse Göttlichkeit« (56) auf, denn Gott selbst habe sie als Instrument auserwählt, um wihn und die anbetungswürdigen Geheimnisse der Religion den Menschen zu zeigen« (57). In einer für Klopstocks Poetik charakteristischen Engführung von literarischen und religiösen Texten wird die Bibel als »das vollkommenste

26 Vgl. Kaiser (Anm. 1), 133-159 (wDer Prophetк), und Wilhelm Große, Studien zu Klopstocks Poetik, München 1977, 130-138.

27 Gotthold Ephraim Lessing, Werke, hrsg. Herbert G. Göpfert, München 1972, II, 307/742 (=15. Brief; ab dem dritten Druck der »Briefe« zu »Ich unsterbliche Seelek verändert).

28 Lessing (Anm. 27), 311 (=16. Brief).

29 Klopstocks lateinische Rede ist zusammen mit einer deutschen Übersetzung Cramers abgedruckt in: C[arl] F[riedrich] Cramer (Hrsg.), Klopstock. Er; und über ihn. Erster Theil. 1724-1747, Hamburg 1780, 54-132; hier: 126.

30 Vgl. Kaiser (Anm. 1), 133-160 (wDer Prophet«). Zum vates-Topos und seinem Fortleben in modemer Dichtung vgl. zuletzt Werner Frick, »Poeta vates. Versionen eines mythischen Modells in der Lyrik der Modernex, in: Matias Martinez (Hrsg.), Formaler Mythos. Beiträge zu einer Theorie ästhetischer Formen, Paderborn 1996, 125-162.

31 Klopstock in Cramer (Anm. 29), $54 \mathrm{f}$. 
Muster des erhabnen und wahrhaftig göttlichen Ausdrucks« (57) bezeichnet; nicht etwa Homer, sondern Moses und die anderen »göttlichen Propheten« (56) erscheinen als Inbegriff des inspirierten Dichters. In einem merkwürdigen Bruch mit dieser Annäherung von Bibel und Dichtung erklärt Klopstock dann jedoch (im Einklang mit der zeitgenössischen Gattungshierarchie), daß sich die »Vortreflichkeit und beynahe Göttlichkeit« (60) der Dichtkunst vor allem in der Gattung des Heldengedichtes, „diesem höchsten Werke der Dichtkunst« (87), zeige (einer Gattung also, die in der Bibel gerade nicht vertreten ist). Das Heldengedicht zeichne sich durch seine "große und herrliche Materie« aus, nämlich durch »eine berühmte Handlung, die, wo nicht den ganzen Erdkreis, doch wenigstens, viele und die gröBten seiner Einwohner angeht, zu besingen, und mit schicklichen und bewunderungswürdigen Erfindungen« ausbilde (61). Deshalb achtet Klopstock "den, der ein Heldengedicht hervorbringt, wie einen himmlischen Genius, andre Poeten aber, die kleinere Gedichte singen, für bloße Menschen« (62). Unter den solcherart ausgezeichneten Verfassern von Heldengedichten sei Milton der Erste, weil er mit der Vollkommenheit der heidnisch-antiken Epiker die, jenen unzugängliche, »himlische Weisheit und Frömmigkeit besingt« (71). Nachdem er Milton so auf den »Gipfel der Größe« (71) gestellt hat, deutet Klopstock jedoch ein noch gröBeres Projekt an, das wohl als verhüllte Ankündigung des eigenen Messias verstanden werden muB: „Du aber, geheiligter Schatten des Miltons! ... zürne nicht über meine Kühnheit, die nicht allein dir $\mathrm{zu}$ folgen, sondern sich auch an einen noch größern und herlichern Stoff zu wagen gedenkt« (75).

In dieser steigernd-kontrastiven Privilegierung von Dichtung (über andere Künste), Heldengedicht (über andere Gattungen), biblischem Heldengedicht (über andere Epentypen), Milton (über andere Epiker) und, in Andeutung, Klopstock selbst (über Milton) wird der Messias in der Pfortenser Abschiedsrede in der skizzierten Hierarchie jeglicher Dichtung an die höchste Stelle gesetzt.

Nichts deutet darauf hin, daß diese Selbstkanonisierung des Messias-Dichters nur Ausdruck vorübergehender Selbstïberschätzung gewesen wäre. Wenige Jahre vor seinem Tod bestätigte Klopstock den in der Abschiedsrede angedeuteten Anspruch, indem er in der Bibliothek seiner Pfortenser Schule eine Prachtausgabe des Messias feierlich neben den Werken der Klassiker aufstellen ließ. Der Schuldirektor Heimbach beschrieb die Zeremonie:

Der Zug gieng dann zur Bibliothek, Die beyden Jünglinge traten ein, das Geschenk der Weihe auf einem Kissen von weißer Seide, mit dem jungen Grün des Waldes geschmückt, tragend. Ein sanfter Gesang ertönte; sie legten es nieder auf dem kleinen darzu errichteten Altar, mit weißer Seide umhangen und mit Immergrün umwunden und am Fuße mit Blumen bestreut. Ein Lorbeerzweig wand sich über die Messiade. ... Mit heiliger Stille stand und sah und horchte die Jugend; und der göttliche Funke schien in aller Herzen sich zu entzünden. ${ }^{32}$

SchlieBlich wären in unserem Zusammenhang auch Klopstocks jahrzehntelang betriebene Projekte zur institutionalisierten Förderung der Künste bis hin zur Ge-

32 Karl Wilhelm Emst Heimbach an Klopstock, 13.4.1800; zit. nach Kevin Hilliard, Philosophy, Letters and the Fine Arts in Klopstock's Thought, London 1987, $19 \mathrm{f}$. 
lehrtenrepublik zu nennen, weil in den geplanten Institutionen stets auch eine Auswahl und Hierarchisierung der Autoren und damit eine Kanonbildung vorgesehen war. ${ }^{33}$

Die Rezeption des Messias vollzog sich teilweise durchaus auf den vom Autor vorgebahnten Wegen. Der prophetisch-inspirierte Gestus wurde von vielen akzeptiert und Klopstock »fast mehr als religiöses denn als ästhetisches Phänomen gewürdigt«.34 Der lebenslange Ehrensold, den Friedrich V. von Dänemark Klopstock ausstellte, galt als angemessene Voraussetzung, damit der Autor sein "von der Vorsehung« verliehenes »Amt, den Messias zu schreiben«, ungehindert ausüben könne (so 1749 der Berliner Hofprediger August F. W. Sack). ${ }^{35}$ Und auch die Gegner des Messias reagierten auf diesen religiösen Geltungsanspruch, wenn sie - wie hier z.B. Johann Christoph Gottsched 1752 - Klopstock vorwarfen, er würde »die Wahrheit mit Lügen verbrämen, und die Geheimnisse des Glaubens zu Legenden machen: die soviel unreinen Menschenwitz in sich halten, daB die ungeheuren Phantasien der Dichter, das Licht der göttlichen Wahrheit wo nicht gar ersticken, doch gewiß umnebeln und verdunkeln müssen«. 36

Die heftige theologische Auseinandersetzung um den Messias war jedoch für das Mißlingen seiner Kanonisierung vermutlich weniger wichtig als ein anderer Sachverhalt, den ich mit einem Rezeptionsbericht aus Goethes Dichtung und Wahrheit illustrieren möchte. Er betrifft den Frankfurter Kaufmann Johann Caspar Schneider, der Ende der 1750er Jahre in Goethes Elternhaus verkehrte.

Auf diesen geschäftstätigen Mann, welcher wenig las, hatte der >Messias‘ gleich bei seiner Erscheinung einen mächtigen Eindruck gemacht. Diese so natiirlich ausgedrückten und doch so schön veredelten frommen Gefühle, diese gefallige Sprache, wenn man sie auch nur für harmonische Prosa gelten ließ, hatten den übrigens trockenen Geschäftsmann so gewomnen, daß er die zehn ersten Gesänge, denn von diesen ist eigentlich die Rede, als das herrlichste Erbauungsbuch betrachtete, und solches alle Jahre einmal in der Karwoche, in welcher er sich von allen Geschäften zu entbinden wußte, für sich im stillen durchlas und sich daran fürs ganze Jahr erquickte. 37

Die Messias-Rezeption des Kaufmanns Schneider (es lieBen sich weitere Zeugnisse dieser Art anführen) erfüllt geradezu idealtypisch Merkmale der sogenannten intensiven Lektüre:38 Schneider liest selten und wiederholt stets

33 Vgl. Kevin Hilliard, »Klopstocks Tempel des Ruhmsк, in: ders., Katrin Kobl (Hrsg.), Klopstock an der Grenze der Epochen, Berlin, New York 1995, 221-239.

34 Kaiser (Anm. 1), 160.

35 Zit. nach Paul Großer, Der Junge Klopstock im Urteil seiner Zeit, Würzburg 1937, 43.

36 Zit. nach Großer (Anm. 35), 57. Ich kann hier nicht auf die Verschiebung von einer wheiligen Poesiek zu einer »Heiligung der Poesiek in Klopstocks eigenen, poetologischen Texten eingehen; vgl. dazu GroBe (Anm. 26), 107-123.

37 Johann Wolfgang Goethe, Werke, hrsg. Erich Trunz, 10. Aufl., München 1981, IX, 80.

38 Vgl. Erich Schön, Der Verlust der Sinnlichkeit oder Die Verwandlungen des Lesers. Mentalitätswandel um 1800, Stuttgart 1987. Schöns plausible Kritik an Rolf Engelsings Bezeichnungen sextensive und sintensive Lektïre، (die ich hier der Kürze halber dennoch verwende) dort 298-300. Zur zeitgenössischen Klopstock-Rezeption vgl. Richard Alewyn, »Klopstocks Leser火, in: Bernhard Fabian (Hrsg.), Festschrift für Rainer Gruenter, Heidelberg 1978, 100-121. 
denselben Text. Die Lektüre findet nicht in der Freizeit des Alltags, sondern in einer feierlichen Zeitspanne während der Karwoche statt. Das Werk dient nicht der Unterhaltung (und wohl auch nicht der Belehrung), sondern der Erbauung.

Andere Dokumente der zeitgenössischen Messias-Rezeption lassen auch Züge einer für die intensive Lektüre typischen sautoritativen Rezeptionssituation< erkennen, in der ein durch den singulären Status seines Textes autorisierter Vortragender einer sozial heterogenen, aber in der Aufführungssituation gemeindeartig zusammengeschlossenen Zuhörerschaft im Dienste einer kollektiven Sinnbildung und -stabilisierung gegenübertritt. ${ }^{39}$ So berichtet Christian F.D. Schubart von Vortragsreisen, auf denen er den Messias vortrug:

Hohe und Niedre, Geistliche und Weltliche, Katholische und Lutherische kamen mit Messiaden unterm Arm in die Vorlesung. $O$ das war ein festlicher Anblick, wie alles so in feyerlicher Stille da saB, wie die Empfindung auffuhr, und in Verwunderung und Thränen ausbrach. Klopstock! Klopstock! scholl's von allen Lippen, wenn eine Vorlesung geendigt war. ... von Jugend auf lemt' ich Declamation aus dem Messias und habe sie [sic] schon in Aalen, Nördlingen, Nürnberg, Erlang, EBlingen, GeiBlingen, Mannheim, München, sonderlich Ludwigsburg beynah unzähligemal vorgelesen. In Ludwigsburg sind Handwerksleute, die den Messias statt eines Erbauungsbuches brauchen, und nach der Bibel (wie's denn auch wahr ist) kein göttlicheres Buch kennen, als dieB. ... F. in R. druckt jezt den Messias und die geistlichen Lieder, und weg gehen sie, zwar nicht, wie Brod zur Zeit der Hungersnoth, reissend und stürmend, aber doch wie tägliches Brod.40

Klopstock selbst trug 1750 aus dem 5. Gesang seines Messias in einem Kloster nahe Zürich vor. Die Nonnen sstanden dicht um mich herum. Ich las, und ich sahe nicht wenig Thränen. ... Sie verstïnden alles, alles, sagten sie; vorher hätten sie nicht alles verstanden«. So überwältigend war die Wirkung des Vortrags, $\mathrm{daB}$ sogar der Beginn der Gebetsstunde vergessen wurde. ${ }^{41}$,Verstehen $<$ ist hier offenbar an die laute Deklamation und die Präsenz des Autors gebunden; der Text allein genügt nicht. Solche Zeugnisse für die überwältigende Wirkung des Messias lassen sich in fast beliebiger Zahl anhäufen. In den 1750er Jahren schien Klopstock unwiderruflich zum deutschen Homer, zum Klassiker zu Lebzeiten aufgestiegen zu sein. 42

39 Vgl. Schön (Anm. 38), 194-197.

40 Schubart an Klopstock (nach dem 22. Mai 1776), in: Friedrich Gottlieb Klopstock, Briefe 1776-1782. Bd. 1: Text, Werke und Briefe, hist.-krit. Ausg., Abt. Briefe, Vu/1, hrsg. Helmut Riege, Berlin, New York 1982, $30 \mathrm{f}$.

41 Klopstock an Denis, 6.1.1767, in: Briefe 1767-1772. Bd. 1: Text, Werke und Briefe, hist.-krit. Ausg., Abt. Briefe, V/1, hrsg. Klaus Hurlebusch, Berlin, New York 1989, $1 \mathrm{f}$.

42 Lessing erklärt Klopstock (allerdings wohl nicht ohne Ironie) im 7. Literaturbrief zum deutschen Homer (Lessing [Anm. 27], V, 42); einen erstarten Fall von Kanonisation beschreibt natürlich sein Sinngedicht "Wer wird nicht einen Klopstock loben? / Doch wird ihn jeder lesen? - Nein. / Wir wollen weniger erhoben, / Und fleißiger gelesen sein« $(I, 9)$. 
III.

Dantes Commedia ist heute obligatorischer Unterrichtsstoff jeder höheren italienischen Schule. Im internationalen Universitätsbetrieb gehört sie zum Klassikerkanon, ja zum »very center of the Canon «43; ihre Anregungskraft für die literarische Produktion bleibt auch im 20. Jahrhundert ungebrochen.44

Der Messias hingegen ist nach wenigen Jahren emphatischer Rezeption aus dem literarischen BewuBtsein verschwunden. Es erscheint mir naheliegend, die nachlassende Rezeption des Messias in der zweiten Hälfte des 18. Jahrhunderts - bei anhaltender Attraktivität der Commedia - (auch) mit dem Übergang von der intensiven zur extensiven Lektüre zu erklären, der sich zur selben Zeit vollzog und bis heute bestimmend geblieben ist. Allerdings bedarf diese Hypothese einiger Erläuterungen.

Es wäre wohl nicht angemessen, den Niedergang des Messias schlichtweg mit seinem religiösen, im Zuge der Säkularisierung obsolet gewordenen Geltungsanspruch zu erklären. Zwar ist ein Aspekt des veränderten Leseverhaltens in der zweiten Jahrhunderthälfte (von den umfassenderen kulturellen Verschiebungen ganz zu schweigen) der (relative, nicht absolute) Rückgang von religiöser Literatur bei gleichzeitiger Zunahme belletristischer Titel; zwischen 1740 und 1800 kehrt sich der relative Anteil von Erbauungsliteratur und Titeln aus dem Bereich der >Schönen Künster an der Gesamtproduktion geradezu um (die absoluten Zahlen für erbauliche Titel verringern sich allerdings nicht). ${ }^{45}$ Aber die von mir hervorgehobenen Merkmale von Klopstocks (quasi-)sakraler Selbstkanonisierung sind ja seither aus der Literatur nicht verschwunden. Die Wahl eines religiösen Erzählstoffs, der Sprechgestus eines poeta vates, der Anspruch auf Sinnstiftung, die Abweisung eines fiktionalisierenden Textverständnisses, Rezeptionsschemata, die das literarische Werk in einen kollektiven, auBeralltäglichen und rituellen Rahmen stellen und eine quasi-religiöse Autorverehrung vorsehen - das alles trifft ja auch für die trotzdem erfolgreiche Commedia zu und hat sich auch nach Klopstock immer wieder (wenngleich vereinzelt) im Literaturbetrieb etablieren können. Jedoch hat sich diese Art der Inszenierung von Literatur mit dem ihr eigenen Rezeptionsschema der sintensiven Lektürer spätestens seit der Romantik auf die Gattung der Lyrik konzentriert; längere narrative Texte fallen eher unter das Schema der sextensiven Lektiures.

Wenn diese Beobachtung zutrifft, dann läßt sich aus ihr eine Hypothese für den unterschiedlichen Erfolg der Commedia und des Messias ableiten. Beide Werke fundieren ihren kanonischen Geltungsanspruch nicht autonomieästhetisch, sondern religiös. Aber Dantes Text war weitaus besser als der Messias an die Be-

43 Harold Bloom, The Western Canon, New York 1994, 89.

44 Vgl. zuletzt Peter Kuon, Lo mio maestro e'l mio autore. Die produktive Rezeption der ,Divina Commedia in der Erzählliteratur der Moderne, Frankfurt a. M. 1993.

45 Vgl. Schön (Anm. 38), 44. Den durchaus komplexen Prozeß der Etablierung fiktionalisierender Lektiire im Laufe des 18. Jahrhunderts schildert Christian Berthold, Fiktion und Vieldeutigkeit. Zur Entstehung moderner Kulturtechniken des Lesens im 18. Jahrhundert, Tübingen 1993. 
dingungen eines modernen Literatur- und Fiktionalitätsbegriffs und an die Erwartungen eines rextensiven، Rezeptionsschemas zu adaptieren. Sein fiktiver, spektakulärer Erzählstoff, die proto-fiktionale Ich-Erzählhaltung, die Plastizität des Stils ermöglichen eine Lektüre der Commedia als belletristischen Text, was nicht der Intention des Autors, wohl aber Lesenormen des modernen Lesepublikums entspricht. So hebt z.B. T.S. Eliot am Autor der Commedia hervor, er sei "extremely easy to read« und »the most universal of poets in the modem languages«, weil seine unbestrittene intellektuelle Schwierigkeit von poetischer Durchsichtigkeit (»lucidity«) begleitet werde. ${ }^{46} \mathrm{Klopstock}$ hingegen setzt seinen Stoff beim Leser eher voraus, als daß er ihn erzählerisch entwickeln würde - die "Erdichtung«, schreibt etwa Klopstock in der Gelehrtenrepublik, »ist keine wesentliche Eigenschaft eines Gedichts «47; insbesondere aber ist der Messias durch einen dunklen Stil geprägt, dessen amimetische Poetik der »Wortbewegung" durchaus die modeme Weiterentwicklung der Lyrik beeinflussen konnte, ${ }^{48}$ aber als fiktionale Erzählliteratur nicht rezipierbar war.

Damit läuft meine Antwort auf die Frage, warum Dantes Commedia heute kanonisch ist, Klopstocks Messias aber nicht, auf einen Kanonbegriff hinaus, der als notwendige (wenngleich nicht hinreichende) Bedingung für Kanonizität die Adaptierbarkeit eines Textes an veränderte Auffassungen und Funktionen von Literatur vorsieht. 46 Thomas] S[tearns] Eliot, „Dante火, in: ders., Selected Essays, London 1969, 237-279,
hier: 238.

47 Friedrich Gottlieb Klopstock, Die deutsche Gelehrtenrepublik. Bd. 1: Text, Werke und Briefe, hist.-krit. Ausg., VII/1, hrsg. Rose-Maria Hurlebusch, Berlin, New York 1975,
171 .

48 Vgl. zuletzt Winfried Menninghaus, »Klopstocks Poetik der schnellen >Bewegung««, in: Friedrich Gottlieb Klopstock, Gedanken über die Natur der Poesie. Dichtungstheoretische Schriften, hrsg. Winfried Menninghaus, Frankfurt a. M. 1989, 259-361. 